\title{
Secretion of Indoleamine 2,3-Dioxygenase, an Immunomodulatory Substance, by Adipose-Derived Mesenchymal Stem Cell
}

\author{
Dian R. Laksmitawati. ${ }^{*}$, Caroline Tan Sardjono ${ }^{2}$, Jeanne A.Pawitan ${ }^{3}$, Mohammad \\ Sadikin $^{3}$, Ferry Sandra ${ }^{4}$ \\ ${ }^{1)}$ Faculty of Pharmacy, Pancasila University, Jakarta, Indonesia ${ }^{2)}$ Faculty of Medicine, Maranatha Christian \\ University, Bandung, Indonesia. ${ }^{3}$ )Faculty of Medicine University of Indonesia, Jakarta, Indonesia ${ }^{4}$ Stem Cell and \\ Cancer Institute, Jakarta, Indonesia
}

\begin{abstract}
Lipoaspirate, a wasted by product from liposuction procedure recently has been shown to contain abundant adipose-derived-mesenchymal stem cells (MSCs). Mesenchymal stem cells (MSCs) have been studied in many research areas to regenerate many cell lineages. In addition, MSCs have immunomodulatory effect. This capability has been utilized in several clinical studies in hematopoetic stem cell and organ transplantation as a strategy to reduce the risk of Graft versus Host Disease (GvHD). It has been reported that the 'stimulated' MSC is able to secrete substances to suppress tissue rejection. One of the substances was known to be indoleamine 2,3-dioxygenase (IDO). A previous study has characterized the secretion of IDO by bone marrow-derived MSCs stimulated by an inflammatory mediator interferon gamma (IFN- $\gamma$ ). IDO has been detected using Western blot analysis and by High Performance Liquid Chromatography (HPLC) assay. The aim of this study was to detect the presence of IDO in AD-MSCs culture with and without INF $\gamma$ stimulation. Our study showed that AD-MSC stimulated with IFN- $\gamma$ significantly secreted high level of IDO as detected by Enzyme-Linked Immuno Sorbent Assay (ELISA). Despite its property as a proinflammatory mediator, IFN- $\gamma$ has shown to be able to induce IDO secretion in MSC culture which suggests the immuno modulatory effect of MSC. This study clearly demonstrates the potential application of adiposederived MSC in the immunomodulatory strategy for allogenic transplantation.
\end{abstract}

Keywords: mesenchymal stem cell, indoleamine 2,3-dioxygenase, IFN- $\gamma$

\section{INTRODUCTION}

Mesenchymal stem cells (MSCs) comprise a population of multipotent progenitor cells capable of both supporting hematopoiesis as well as to differentiate into many tissue lineages. In the appropriate microenvironment, MSCs differentiate into various cell types, including cells originated from mesodermal lineage (adipocytes, osteoblasts, chondrocytes, and cardiomyocytes), into endoderm-derived cells (hepatocytes) and ectoderm-derived cells (neurons)(Sanchez-Ramos et al., 2000; Schwartz et al., 2002; Zhang et al., 2008). Recently utilization of MSCs have been reported to prevent severe graft-versus-host disease (GvHD) in allogenic transplantation (Le Blanc et al., 2004). Mesenchymal stem cells have low immunogenicity and are capable of inhibiting allogenic T-cell responses(Le Blanc, 2006). MSCs may evade the detection and elimination by the immune system, and suppress alloreactivity through modulation of most major immune cell activities. However, the exact mechanism of the immunomodulatory effects of MSCs is not completely understood. Both direct and indirect effects have been suggested through either cell-cell interaction or soluble factors that create a local immunosuppressive environment (Le Blanc and Ringden, 2007).

*Corresponding author e-mail: dianratih.ffup@gmail.com 
Several compounds such as hepatocyte growth factor (HGF), transforming growth factor (TGF- $\beta 1$ ) (Di Nicola et al., 2002), IL-10, IL6, prostaglandin E2 (Aggarwal and Pittenger, 2005), nitric oxide (Sato et al., 2007) and indoleamine 2,3-dioxygenase (IDO) (Meisel et al., 2004) secreted by MSCs have been reported to involve in MSCs immunomodulatory effect .

There are several sources of MSCs i.e. bone marrow, umbilical cord blood, peripheral blood and adipose tissue. Human Adipose Derived-stem cells (hADSCs) share unique features of its counterpart in bone marrow, such as differentiation potential, low immunogenicity and the ability to suppress immune response (Puissant et al., 2005; Yanez et al., 2006)

IDO is an apoenzyme that is encoded by a single gene with 10 exons in human chromosome 8 (Suzuki et al., 2003). Various cell types, including certain myeloid-lineage cells (monocyte-derived macrophages and dendritic cells), fibroblasts, endothelial cells and some tumour-cell lines, express IDO after exposure to IFN- $\gamma$. (REFF) IDO activity is found constutively at maternal-fetal interface, and is expressed by human extravillous trophoblast cells. Outside placenta, functional IDO expression was reported to be highest in the mouse epididymis, gut, lymph nodes, spleen thymus and lungs (Mellor and Munn, 2004). The presence of constitutive and inducible IDO expression in these tissues might function as an antiinflammatory/immunosuppressive mechanism, (Mellor 2004) by initiating the degradation of tryptophan along the kynurenine pathway. Some of tryptophan metabolites suppress $\mathrm{T}$ cell proliferation in vitro or cause $\mathrm{T}$ cell apoptosis and some can affect NK cell function (Munn and Mellor, 2007).

A previous study has characterized the secretion of IDO by bone marrow-derived MSCs stimulated by an inflammatory mediator interferon gamma (IFN $\gamma)$. (REFF) IDO has been detected using Western blot analysis and High Performance Liquid Chromatography (HPLC) assay. The aim of this study was to detect the presence of IDO in AD-MSCs culture with and without INF- $\gamma$ stimulation by Enzyme-Linked Immuno Sorbent Assay (ELISA).

\section{METHODS}

\section{Mesenchymal stem cell isolation from lipoaspirates}

Protocols in this study have been reviewed and approved by the Stem Cell and Cancer Institute Institutional Review Board prior to the study (number 13/IRB/SCI/KF/2008 and 19/IRB/SCI/KF/20102. Lipoaspirates, which were discarded waste materials from individuals who experienced tumescent liposuction surgery were obtained from hospitals in Jakarta. Lipoaspirates were stored at $2-8^{\circ} \mathrm{C}$ for no longer than 24 hours before they were used. The methods used to isolate the MSCs from lipoaspirate were adapted from previously reported (Sardjono et al., 2009; Suzuki et al., 2001) The raw lipoaspirates were washed 3 times by diluting them with equal volume of Phosphate Buffer Saline (PBS). The diluted lipoaspirates were centrifuged at $430 \times \mathrm{g}$ for 10 minutes without brakes at $20^{\circ} \mathrm{C}$. After centrifugation, the target cell-containing lipid phase was removed from the top and transferred in to new tubes and diluted with an equal volume of PBS. The next step was digesting the cellcontaining lipid fraction with an equal volume of pre-warmed $\left(37^{\circ} \mathrm{C}\right) \quad 0.075 \%$ collagenase type I (Sigma) in PBS. Enzyme digestion was done by incubation at $37{ }^{\circ} \mathrm{C}$ for 30 minutes on an orbital shaker. After digestion, enzyme activity was neutralized by adding an equal volume of Dulbecco's modified Eagle's medium (DMEM) containing $10 \%$ Fetal Bovine Serum (FBS). Digested product was then subjected to centrifugation at $600 \times \mathrm{g}$ for 10 minutes. The pellet was resuspended in DMEM with $10 \% \mathrm{PBS}$, and then filtered through a $100-\mu \mathrm{m}$ strainer mesh. The collected cells after filtration were then ready for utilization. An aliquot was taken for cell count using hemocytometer under a light microscope to determine cell yields. Viable cell count was determined with a hemocytometer and the trypan blue dye exclusion technique.

\section{MSCs culture}

The simplest technique to purify MSC from other contaminating cells was done by allowing the cells to adhere on plastic-surfaced disc. Cells were seeded at $1.3 \times 10^{5}$ cells $/ \mathrm{cm}^{2}$ in MesenCult complete medium (StemCell Technologies 05401 and 05402), $100 \mathrm{unit} / \mathrm{ml}$ penicillin and $0.1 \mathrm{mg} / \mathrm{ml}$ streptomycin (Sigma P0781), then kept in $37^{\circ} \mathrm{C}$, $5 \% \mathrm{CO}_{2}$. After 2-3 days unwanted cells (nonMSCs and debris) were removed by two washes with medium, and MSCs were expanded to reach $80 \%$ confluence. In subsequent 6-7 days, adherent cells were detached using $0.25 \%$ trypsin EDTA solution (Sigma T4049), then DMEM medium + $20 \%$ FBS was used to inactivate the trypsin. Detached cells with fibroblast-like morphology were cultured in another flask with cell density of $1.2 \times 10^{5}$ cells $/ \mathrm{cm}^{2}$ for 1 week or until confluence was achieved. For IFN- $\gamma$ stimulation, cells were 
seeded at 5000 cells $/ \mathrm{cm} 2$ in Mesencult medium and were incubated until the cells were attached (1 hour). Interferon $\gamma$ was added to the culture. We use IFN- $\gamma$ 10, 50, 100 and $200 \mathrm{IU} / \mathrm{ml}$ medium. The supernatants were collected in the sixth day of culture and kept in $-80^{\circ} \mathrm{C}$ after sentrifugation to separate the floating dead cells.

\section{Flowcytometry assay to determine MSC homogenous population}

To confirm the quality of the MSCs isolated, several assays were conducted. We use positive (CD105, CD73) and negative MSCs markers (HLA-DR, CD 14, CD 19, CD 45 and CD 34). Cells were harvested and stained with the appropriate surface monoclonal antibodies (PE conjugated: CD105 (abcam 53321-100), CD73 (BD550257), HLA-DR (abcam 23901), CD 19 (abcam 1168-500); FITC-conjugated CD14 (abcam 28061-100), CD45 (BD 555482) and CD 34 (BD 348053)) following manufacturer's instructions. Isototype controls ( $\mathrm{mIgG1}$ (BD349041 and BD 349043), and mIgG2 (BD349053)) were used to determine background staining. After washing, the cells were fixed, and 5000 events were acquired using a FACS Calibur 3 argon laser 488nm (Becton Dickinson). CellQuest-pro software was used for acquisition and analysis.

\section{ELISA methods to measure IDO}

IDO was measured with ELISA kit (E1547Hu, Uscn Life Science Inc.Wuhan). The microtiter plate was pre-coated with an antibody specific to IDO. Standards $(0-100 \mathrm{IU} / \mathrm{mL})$ or samples were then added to the appropriate microtiter plate wells followed by a biotinconjugated polyclonal antibody specific for IDO. Next avidin conjugated to Horseradish Peroxidase (HRP) was added to each microplate well and incubated for 30 minutes. A tetramethylbenzidin (TMB) substrate solution was added to each well. The presence of IDO in the samples was detected by biotin-conjugated antibody and enzyme- conjugated avidin, the enzyme-substrate reaction was terminated by addition of a sulphuric acid solution and the color change was measured spectrophotometrically at wavelength of $450 \mathrm{~nm}$. Standard curve with known IDO concentration was generated using $100,50,25,12.5,6.25,3.12,1.56$, 0 (blank) IU/mL. The concentrations of IDO in the samples were determined by projecting the samples reading in the standard concentration curve.

\section{RESULTS}

\section{Morphology and surface markers of mesenchymal stem cells}

Using the described methods, MSCs were able to be obtained from lipoaspirate. In cell culture, the isolated cells showed a fibroblast-like morphology, which is characteristic for MSCs (Figure 1). Contaminant cells could be eliminated by passaging cells. High purity MSCs could be achieved after the second passage. To confirm the purity of MSCs we used some of the suggested markers according to ISCT (Dominici et al., 2006) and measured the purity by flow cytometer.

Cell surface molecules expressions were determined by flowcytometry assay. Based on standard criteria for defining multipotent mesenchymal stem cells, we characterized the cultured cells using markers for CD105 (known as endoglin), and CD73 (ecto 5'-nucleotidasethat is recognized by $\mathrm{SH} 3$ and $\mathrm{SH} 4$ ). We confirmed our isolated and cultured cells were MSCs as they mainly expressed CD105 and CD 73, but did not HLA-Class II, CD 45, CD 34, CD14, and CD19 (Figure 2), which was consistent with the profile of MSCs. In this study, the flowcytometry gated results for CD 105, CD 73, CD34, CD 45, were $92.04 \%, 99.68 \%, 0.07,0.22 \%$ respectively and CD14, 19 and HLA-DR were $0 \%$. 


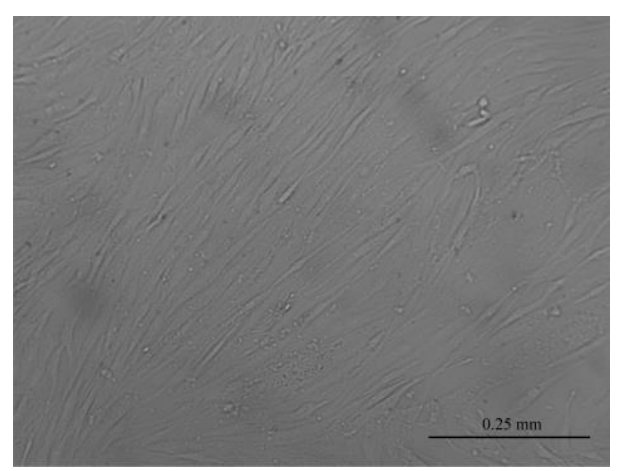

Figure I. ADSCs showed fibroblast-like morphology
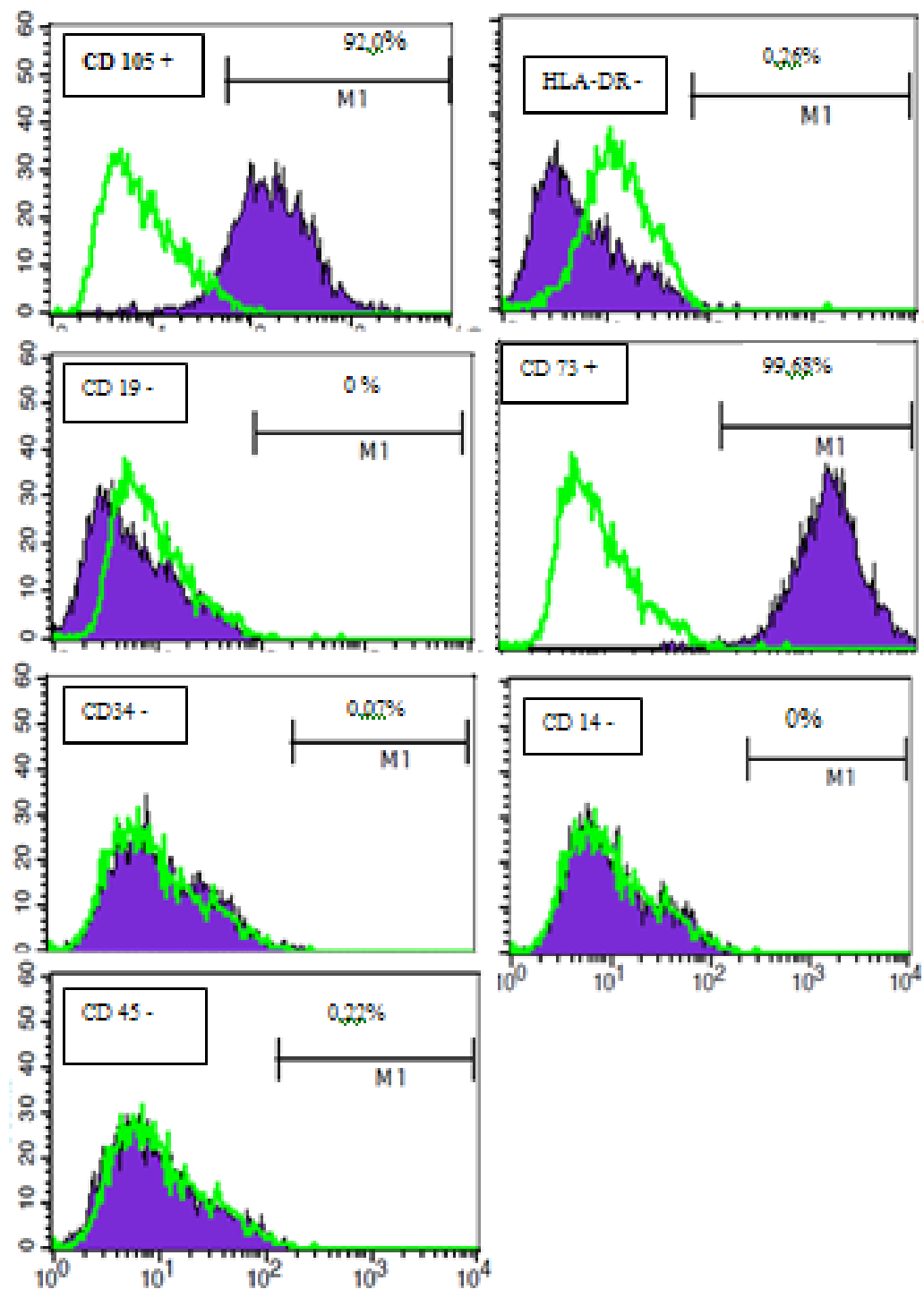

Figure 2. Flowcytometry analysis of molecules expression on MSC from lipoaspirate. Flowcytometry assay showed the cultured cells were consistent with the characteristic of MSC, i.e. CD 105 and CD73 were positive, while CD- 45, CD34, CDI9 , CDI4 and HLA-DR were negative (< I\%). 


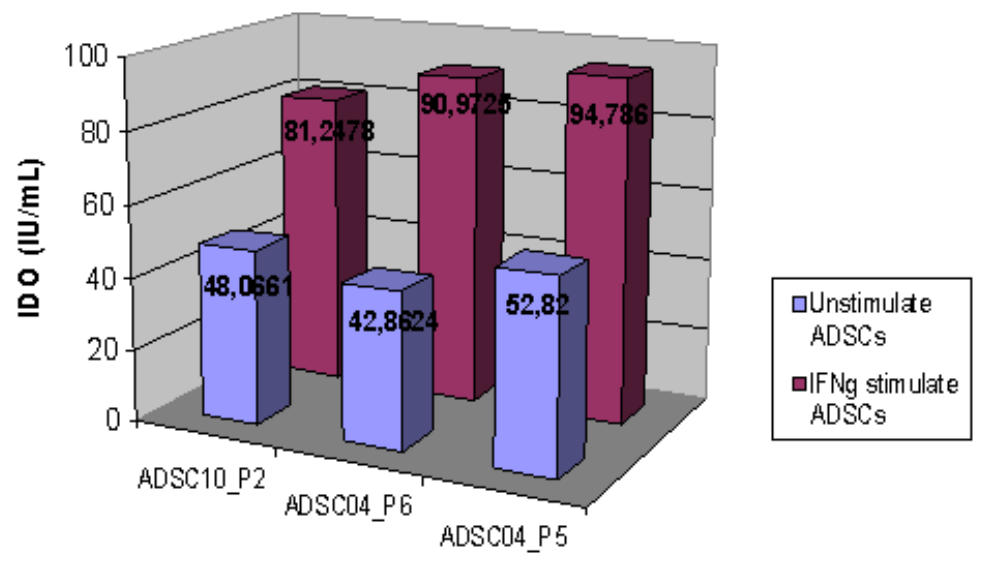

Figure 3. IDO level $(I \mathrm{U} / \mathrm{mL})$ in ADSC culture with passage varian. Using lipoaspirate from 2 different donors: ADSC 10 and ADSC 04.

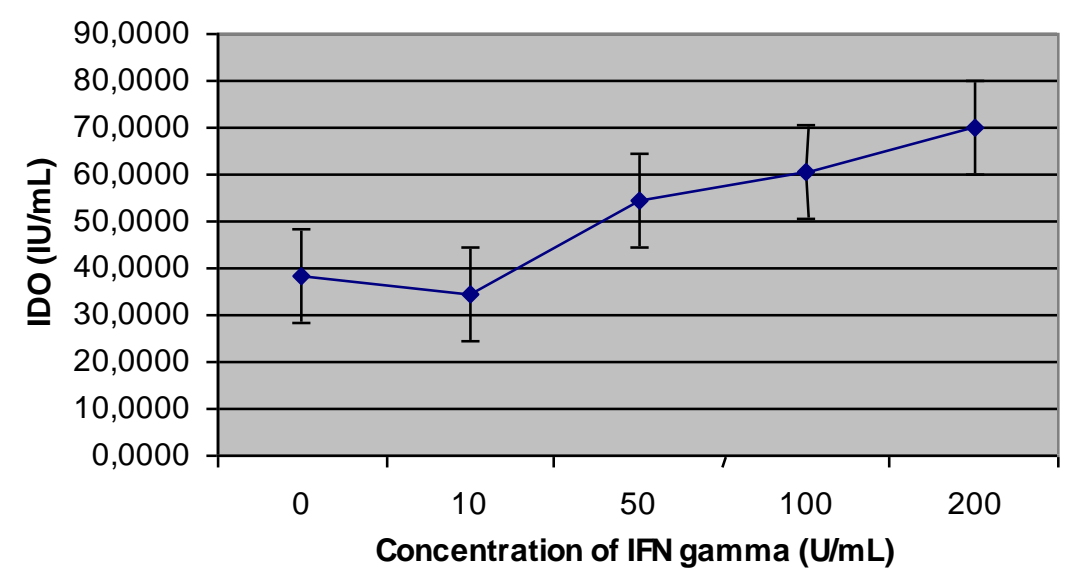

Figure 4. Secreted IDO in the culture with and without IFN- $\gamma$ stimulation by ELISA.

\section{IDO level in AD-MSCs}

In this study, AD-MSCs samples from 2 donors and different passages showed about the same IDO level. The level range was 42.86-52.82 $\mathrm{IU} / \mathrm{mL}$ (Figure 3) The quantity of secreted IDO was higher when the culture was stimulated with IFN- $\gamma \quad(81,25-94,79 \quad \mathrm{IU} / \mathrm{mL})$ (Figure 4). The secreted IDO level showed a positive association trend with the increasing IFN- $\gamma$ level by culturing ADSC for 6 days.

\section{DISCUSSION}

MSC can be isolated from adipose tissue. Here we isolated MSC from liposuction, which was a waste from surgery. With a modified simple protocol adopted from Zuk et al. (2009) and Sardjono et al. (2009) we could get MSCs. The purity of MSC devoid of hematopoietic cells could be achieve after passage 2 . This cells might be an alternative source of MSCs beside bone marrowderived cells.

The use of MSCs in regenerative medicine is of great interest and represents a major goal to achieve in the coming years. The interest resides in their ability to be easily isolated and expanded $e x$ vivo and multipotential differentiation capabilities together with their immunosuppressive properties. Soluble factors such HGF, PGE2), TGF- $\beta 1$, IDO, nitric oxide and IL-10 have been implicated. Several reports have also shown that inflammatory cytokines such as TNF- $\alpha$ and IFN- $\gamma$ may regulate the MSC mediated immunosuppresion (DelaRosa et al., 2009). Several studies have measured IDO in MSC culture. By different method in this study, we analyzed IDO level by ELISA method in ADSC culture stimulated with and without IFN- $\gamma$.

This study showed that IDO was secreted to the supernatant of ADSC culture with or without IFN- $\gamma$ stimulation. The quantity of secreted IDO in 
culture with IFN- $\gamma$ was higher compared to culture without IFN- $\gamma$ addition. Several studies showed that IFN- $\gamma$ was a prerequisite for IDO secretion. The difference found in our result might be due to the fact that we did not measure IDO level in the medium. As we used ELISA kit to detect IDO, the antibody might react with IDO-like proteins in the medium. Therefore IDO level in unstimulated culture can be regarded as baseline.

DelaRosa et al, 2009 measured IDO activity by determining both Trp and Kyn level in conditioned supernatant from ADSC culture using HPLC They found that IDO activity was induced by IFN- $\gamma$ stimulation. Further, IDO activity was significantly reduced when IFN- $\gamma$ signaling was blocked with anti bodies. A study on IDO gene inserted hADSC clone showed that hADSC-IDO cells secreted the active enzyme constutively, which resulted in increased Kyn level in the medium. The IDO activity was further increased by IFN- $\gamma$ stimulation. The hADSC without inserted IDO gene did not show an increase in Kyn level in the medium after 48 hours of culture.(DelaRosa et al., 2009)

Meisel et al, (2004), detected the secretion of IDO by Western blot method, and measured the IDO activity by photometric determination of kynurenin concentration in the supernatant. After 3 days, bone marrow stem cells (BMSCs) with and without IFN- $\gamma$ stimulation were harvested and subjected to Western blot analysis using IDOspecific monoclonal antibody. Human BMSC secreted IDO only upon stimulation with IFN- $\gamma$ at a concentration of $500 \mathrm{U} / \mathrm{mL}$. They showed that hBMSCs expressed functional IDO activity upon stimulation with IFN- $\gamma$. The higher the concentration of IFN $-\gamma$ the greater the IDO activity (Meisel et al., 2004)

This result is in line with our data. Our data showed positive association trend of between IFN $-\gamma$ and IDO level that was measured by ELISA. Our data showed that IDO could be detected in ADSC culture without addition of IFN- $\gamma$. However IFN- $\gamma$ addition caused e higher IDO secretion. This result differed from the result of a recent study. There are many factors that may cause the difference between our result and the recent study, such as the methods of IDO detection, period of culturing, source of MSC, media and the concentration of IFN- $\gamma$ for the stimulation.

Interferon gamma is a proinflammatory mediator, but in MSC culture, it behaves differently, because INF $\gamma$ stimulates IDO secretion that has an immunomodulatory effect. This study clearly demonstrates the potential application of
AD-MSC in the immunomodulatory strategy for allogenic transplantation.

\section{CONCLUSION}

By detecting IDO with ELISA method in the supernatant of a 6 day AD-MSCs culture, we found that IDO could be secreted. Addition of IFN- $\gamma$ resulted in higher secretion of IDO.

This study clearly demonstrates the potential application of AD-MSCs in the immunomodulatory strategy for allogenic transplantation.

\section{ACKNOWLEDGMENT}

The authors want to thank Boenjamin Setiawan, dr., PhD. (founder of Stem Cell and Cancer Institute) who supported this research, to donors for donating their lipoaspirate and the collaborating hospital staffs who have coordinated the collection of materials to be used in this study.

\section{REFERENCES}

Aggarwal, S., and Pittenger, M.F., 2005. Human mesenchymal stem cells modulate allogeneic immune cell responses, Blood, 105(4), I8I5-1822.

DelaRosa, O., Lombardo, E., Beraza, A., Mancheno-Corvo, P., Ramirez, C., Menta, R., Rico, L., Camarillo, E., Garcia, L., Abad, J.L., Trigueros, C., Delgado, M. and Buscher, D., 2009, Requirement of IFNgamma-mediated indoleamine 2,3dioxygenase expression in the modulation of lymphocyte proliferation by human adipose-derived stem cells, Tissue Eng Part A, I 5(I0), 2795-2806.

Di Nicola, M., Carlo-Stella, C., Magni, M., Milanesi, M., Longoni, P.D., Matteucci, P., Grisanti, S. and Gianni, A.M., 2002, Human bone marrow stromal cells suppress Tlymphocyte proliferation induced by cellular or nonspecific mitogenic stimuli, Blood, 99(10), 3838-3843.

Dominici, M., K. Le Blanc, I. Mueller, I. SlaperCortenbach, F. Marini, D. Krause, R. Deans, A. Keating, D. Prockop, and E. Horwitz. 2006. Minimal criteria for defining multipotent mesenchymal stromal cells. The International Society for Cellular Therapy position statement. Cytotherapy 8(4), 3I5-3 I7. 
Le Blanc, K., 2006, Mesenchymal stromal cells: Tissue repair and immune modulation, Cytotherapy, 8(6), 559-56I.

Le Blanc, K., Rasmusson, I., Sundberg, B., Gotherstrom, C., Hassan, M., Uzunel, M. and Ringden, O., 2004, Treatment of severe acute graft-versus-host disease with third party haploidentical mesenchymal stem cells, Lancet, 363(94|9), |439-|44|.

Le Blanc, K. and Ringden, O., 2007, Immunomodulation by mesenchymal stem cells and clinical experience, J Intern Med, 262(5), 509-525.

Meisel, R., Zibert, A., Laryea, M., Gobel, U. Daubener, W. and Dilloo, D., 2004, Human bone marrow stromal cells inhibit allogeneic T-cell responses by indoleamine 2,3-dioxygenase-mediated tryptophan degradation, Blood, I03(12), 46I9-462I.

Mellor, A.L. and Munn, D.H., 2004, IDO expression by dendritic cells: tolerance and tryptophan catabolism, Nat Rev Immunol, 4(I0), 762-774.

Munn, D.H. and Mellor, A.L., 2007, Indoleamine 2,3-dioxygenase and tumor-induced tolerance, J Clin Invest, I I 7(5), I I47-I I 54.

Puissant, B., Barreau, C., Bourin, P., Clavel, C., Corre, J., Bousquet, C., Taureau, C., Cousin, B., Abbal, M., Laharrague, P., Penicaud, L., Casteilla, L. and Blancher, A., 2005, Immunomodulatory effect of human adipose tissue-derived adult stem cells: comparison with bone marrow mesenchymal stem cells, $\mathrm{Br} J$ Haematol, I 29(I), I I8-1 29.

Sanchez-Ramos, J., S. Song, F. Cardozo-Pelaez, C. Hazzi, T. Stedeford, A. Willing, T.B. Freeman, S. Saporta, W. Janssen, N. Patel, D.R. Cooper, and P.R. Sanberg. 2000. Adult bone marrow stromal cells differentiate into neural cells in vitro, Exp Neurol, I64(2), 247-256.

Sardjono, C., Setiawan, A.M., Frisca, V. Saputra, G. Aniko, and Sandra, F., 2009, Application of a modified method for stem cell isolation from lipoaspirates in a basic lab, Medical Journal of Indonesia, I 8(2), 91-96.

Sato, K., Ozaki, K., I. Oh, I., Meguro, A., Hatanaka, K., Nagai, T., Muroi, K. and Ozawa, K., 2007, Nitric oxide plays a critical role in suppression of $\mathrm{T}$-cell proliferation by mesenchymal stem cells, Blood, 109, 228234.

Schwartz, R.E., Reyes, M., Koodie, L., Jiang, Y., Blackstad, M., Lund, T., Lenvik, T., Johnson, S. Hu, W.S. and Verfaillie, C.M., 2002, Multipotent adult progenitor cells from bone marrow differentiate into functional hepatocyte-like cells, J Clin Invest, 109, 1291-1302.

Suzuki, S., S. Tone, O. Takikawa, T. Kubo, I. Kohno, and Y. Minatogawa. 200I. Expression of indoleamine 2,3dioxygenase and tryptophan 2,3dioxygenase in early concepti, Biochem $J$ 355(Pt 2), 425-429.

Suzuki, T., Yokouchi, K., Kawamichi, H., Yamamoto, Y., Uda, K. and Yuasa, H.J., 2003, Comparison of the sequences of Turbo and Sulculus indoleamine dioxygenase-like myoglobin genes, Gene 308, 89-94.

Yanez, R., Lamana, M.L., Garcia-Castro, J., Colmenero, I., Ramirez, M. and Bueren, J.A., 2006, Adipose tissue-derived mesenchymal stem cells have in vivo immunosuppressive properties applicable for the control of the graft-versus-host disease, Stem Cells, 24, 2582-259l.

Zhang, R.L., Jiang, E.L., Wang, M., Zhou, Z., Zhai, W.J., Zhai, W.H., Wang, H., Wang, Z.Y., Bao, Y.S., Du, H. and Han, M.Z., 2008, [Differentiation of bone marrow derived from mesenchymal stem cells into cardiomyocyte-like cells induced by coculture with rat myocardial cells], Zhongguo Shi Yan Xue Ye Xue Za Zhi, I6, | | | - I | I5. 